\title{
So, what is chiropractic? Summary and reflections on a series of papers in Chiropractic and Manual Therapies
}

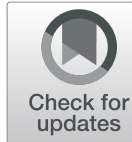

\author{
Jan Hartvigsen ${ }^{1,2^{*}}$ (D) and Simon D. French ${ }^{3}$
}

\begin{abstract}
This commentary brings the 2017-2019 thematic series What is Chiropractic? to a close. The 18 papers published in the series contribute to a better understanding of what chiropractic is, where chiropractors practice and function, who seeks their care, what chiropractors do, and how they interact with other healthcare professionals. Several papers in the series highlighted deeply rooted disagreements within chiropractic about fundamental issues pertaining to ideology, acceptance of scientific evidence as the basis for clinical practice and the future of chiropractic. If the chiropractic profession is to remain relevant in today's evidence-based healthcare environment, there is an urgent for the profession to undertake further research to describe what chiropractic is, what chiropractors do, and provide evidence for the value of these activities to patients and healthcare decision makers.
\end{abstract}

Keywords: Chiropractic, Education, Disability, X-ray, Debate

\section{Background}

In November of 2017, Chiropractic and Manual Therapies issued a call for papers under the common theme "What is Chiropractic?". The motivation for the thematic series was to help define chiropractic better to stakeholders inside and outside of the profession. The need for the thematic series was a perceived confusion about what chiropractic is, what chiropractors do, and how they contribute to the health and well-being of patients [1] in spite of official definitions such as by the World Federation of Chiropractic: Chiropractic is a health profession concerned with the diagnosis, treatment and prevention of mechanical disorders of the musculoskeletal system, and the effects of these disorders on the function of the nervous system and general health. There is an emphasis on manual treatments including spinal adjustment and other joint and soft-tissue manipulation [2]. Two years later, 18 papers have been published in the series, and it is time to take stock and examine what we have learned through this initiative.

\footnotetext{
* Correspondence: jhartvigsen@health.sdu.dk

${ }^{1}$ Department of Sports Science and Clinical Biomechanics, University of Southern Denmark, Campusvej 55, 5230 Odense M, Denmark

${ }^{2}$ Nordic Institute of Chiropractic, Campusvej 55, 5230 Odense M, Denmark Full list of author information is available at the end of the article
}

\section{Main text}

We have grouped the published papers in the thematic series under three headings and summarize them below. An overview of aims and conclusions of papers in this series is provided in Table 1.

\section{Papers describing the chiropractic profession and chiropractic practice}

The overall global view of the chiropractic profession was provided in two articles. Stochkendahl et al. provided a global view of the chiropractic workforce and concluded that chiropractors are present in 90 of 193 United Nations member nations with the highest prevalence in high-income countries [3]. Although chiropractors are legally recognized in $75 \%$ of those countries, and chiropractic treatment is fully or partially subsidized by national healthcare systems in around $50 \%$, in some countries chiropractic does not fall under any law or may even be illegal [3]. Beliveau et al. reviewed 52 studies dealing with utilization of chiropractic and found that, generally, utilization was highly variable and population dependent, with no clear change in utilization of chiropractors globally between 1980 and 2015 [4]. They found that the typical chiropractic patient is seeking care for a musculoskeletal 
Table 1 Aims and conclusions of papers published in the series What is chiropractic?

\begin{tabular}{ll}
\hline First author; year; title; study design & Aim of paper \\
\hline Papers describing the chiropractic profession and chiropractic practice \\
$\begin{array}{ll}\text { Beliveau et al. } 2017 . & \text { To document the current state of knowledge } \\
\text { on the: } 1 \text { ) utilization of chiropractic services; } 2)\end{array}$ \\
$\begin{array}{ll}\text { The chiropractic profession: a scoping review of } & \begin{array}{l}\text { reasons for attending chiropractic care; 3) } \\
\text { utilization rates, reasons for seeking care, patient } \\
\text { profiles, and care provided. }\end{array} \\
\text { patients; and } 4 \text { ) types of chiropractic assessment } \\
\text { and treatment provided worldwide. }\end{array}$
\end{tabular}

Conclusion

scoping review and treatment provided worldwide.

To describe the experiences of chiropractors engaging in sickness absence management (SAM) and to compare and contrast chiropractors' integration of SAM in their model of care in a context with legislated sickness certification rights (Norway) and in two contexts without sickness certification rights (Sweden and Denmark).

\section{Salsbury et al. 2018.}

Be good, communicate, and collaborate: a qualitative analysis of stakeholder perspectives on adding a chiropractor to the multidisciplinary rehabilitation team.

Qualitative analysis based on personal and focus group interviews

Jenkins et al. 2018.

Current evidence for spinal $X$-ray use in the chiro practic profession.

Narrative review

To explore stakeholder perceptions of the qualities preferred in a chiropractor from the perspectives of patients, families, and interdisciplinary team members affiliated with this rehabilitation setting.

To review 1) the current use of spinal X-ray imaging within chiropractic clinical practice; 2) the evidence for potential reasons for obtaining spinal X-rays within chiropractic; 3 ) the evidence of possible risks or limitations associated with the use of spinal X-rays; and 4) guidelines for the appropriate use of imaging in chiropractic clinical practice.

To describe work-related physical acute injuries and overuse complaints as well as psychosocial stress among chiropractors in primary care chiropractic practice in Denmark during the previous year.

overuse complaints, and the psychosocial work environment in Danish primary care chiropractic practice - a cross-sectional study.

Cross-sectional study

Innes et al. 2018

Comparing the old to the new: A comparison of similarities and differences of the accreditation standards of the chiropractic council on education-international from 2010 to 2016. Review of published accreditation standards

Across different countries and regions, the average 12- month utilization rate of chiropractic services was $9.1 \%$ with little change between 1980 and 2015. Musculoskeletal conditions, such as back and neck pain, were the predominant reason for people attending chiropractic care. Typically, chiropractic patients were female, aged 43.4 years, and employed. Four out of five patients who consulted a chiropractor received spinal manipulation; however, chiropractors also commonly provided other treatments including soft-tissue therapy and formal patient education.

Chiropractors with patient management expertise can fulfil a key role in SAM and by extension work disability prevention when these practices are legislatively supported. In cases where these practices occur informally, however, practitioners face systemic-related is sues and professional self-image challenges that tend to hamper them in fulfilling a more inte grated role as providers of WDP practices.

Study participants supported the addition of a chiropractor to the multidisciplinary team who practiced in a safe, evidence-based, patientcentered manner. Interprofessional skills that en hanced teamwork, intrapersonal qualities to support patients' emotional journeys through the rehabilitation process, and an organizational perspective that amplified the mission of the in stitution also were desired.

The use of spinal $X$-rays in chiropractic has been controversial, with benefits for the use of rou tine spinal $X$-rays being proposed by some ele ments of the profession. However, evidence of these postulated benefits is limited or nonexistent. There is strong evidence to demon strate potential harms associated with spinal $X$ rays including increased ionizing radiation ex posure, overdiagnosis, subsequent low-value in vestigation and treatment procedures, and in creased unnecessary costs. Therefore, in the vast majority of cases who present to chiropractors, the potential benefit from spinal $X$-rays does not outweigh the potential harms.

Danish practicing chiropractors commonly reported physical work-related acute injuries or overuse complaints. Overuse complaints are sig nificantly more common in women and occur primarily in the low back, wrist, thumb, and shoulder. Chiropractors with less than five years in practice report more overuse complaints than chiropractors with more than five years in prac tice. Chiropractors in Denmark generally have a good psychosocial work environment.

1. to compare the Council on Chiropractic Education International 2016 Accreditation Standards with their previous 2010 Accreditation Standards, including the way they were developed, and; 2 . to explore similarities and differences of prescribed recommendations to identify any changes to procedures, concepts, and emphases; 3 . to comment on whether
Some positive changes have taken place, such as having bravely moved towards the musculoskeletal model, but on the negative side, the requirement to produce graduates skilled at dealing with scientific texts has been removed. A more robust development approach including better transparency is needed before implementation of CCE standards and evidence-based concepts should 
Table 1 Aims and conclusions of papers published in the series What is chiropractic? (Continued)

\begin{tabular}{ll}
\hline First author; year; title; study design & Aim of paper \\
\hline & $\begin{array}{l}\text { these changes are likely to be for the better or } \\
\text { the worse. }\end{array}$ \\
& \\
Funk et al. 2018 & $\begin{array}{l}\text { To determine the prevalence of the term } \\
\text { subluxation in the course titles or descriptions }\end{array}$ \\
$\begin{array}{l}\text { The prevalence of the term subluxation in } \\
\text { chiropractic degree program curricula throughout } \\
\text { the world. }\end{array}$ & world for which information could be found. \\
Review of websites & \\
Stochkendahl et al. 2019. & $\begin{array}{l}\text { To describe the chiropractic workforce } \\
\text { worldwide in terms of the number of } \\
\text { chiropractors, education, access, reimbursement } \\
\text { schemes, scope of practice, and legal rights. }\end{array}$
\end{tabular}

Cross-sectional survey

Stochkendahl et al. 2019.

Managing sickness absence of patients with musculoskeletal pain - a cross-sectional survey of Scandinavian chiropractors.

Cross-sectional survey

Mior et al. 2019.

Chiropractic services in the active duty military setting.

Scoping review

Wirth et al. 2019

An observational study on trajectories and outcomes of chronic low back pain patients referred from a spine surgery division for chiropractic treatment. Longitudinal cohort study

Axen et al. 2019

Chiropractic maintenance care - what's new? Systematic review
Conclusion

be integrated in the programs. The CCEInternational should consider the creation of a recognition of excellence in educational pro grams and not merely propose minimal standards.

The term subluxation was found in all but two US chiropractic course catalogues. The term was mentioned over eight times more frequently in US than non-US course catalogues. Similarly, subluxation was found greater than nine times more frequently in US course descriptions than in non-US descriptions.

The [chiropractic] profession is represented in 90 countries, but the distribution, chiropractic educational institutions, and governing legislations and regulations largely favour highincome countries. There is a large underrepresentation in low- and middle-income countries in terms of provision of services, edu cation and legislative and regulatory frame works, and the available data from these coun tries are limited.

To 1) determine the prevalence of musculoskeletal practitioners' key practice behaviors, and perceptions and beliefs about SAM using Scandinavian chiropractors; 2) to determine what characteristics of the practitioners, practice behaviors, perceptions and beliefs, and country were associated with two different practice behaviors.

To describe 1) access of chiropractic services; 2) chiropractic scope of practice, e.g. procedures, processes, and actions; 3 ) service model and location; and 4) type of condition treated, duration, and outcomes of treatment provided to active duty military members.

To describe the trajectories and outcomes of patients with chronic LBP referred from the spine surgery division to the chiropractic teaching clinic.

1. To define the concept of Maintenance Care and the indications for its use; 2 . To describe chiropractors' belief in Maintenance Care and patients' acceptance of it; 3 . To establish the prevalence with which chiropractors use Maintenance Care and possible characteristics of the chiropractors associated with its use; 4. To determine its efficacy and cost-effectiveness for various types of conditions.
Whilst not always engaged in sickness absence management (SAM) with regards to musculoskeletal pain, chiropractors favor a 'return-to-work' rather than a 'stay-at-home' approach. Norwegian chiropractors who have the right to prescribe sick leave consistently report more positive perceptions and beliefs with the process compared with the Danish and Swedish chiropractors who do not have these rights. Several practice behaviors and perceptions and beliefs are associated with these outcomes; however, system or organizational barriers are linked to clinician non-engagement.

The majority of the articles emanated from the US and were cross-sectional in nature. Two re cent RCTs provide evidence of comparative ef fectiveness of adding chiropractic care to usual medical care. Despite the reported use of chiro practic services in Australia, Canada, and the US, there is little available published evidence re lated to the nature, use, and outcomes of chiro practic care in active duty military.

Chronic LBP patients with long-lasting pain, reduced general health and high bio-psychosocial impairment, referred from spine surgery to a chiropractic teaching clinic, benefit from being co-managed by surgeons and chiroprac tors. Present pain intensity and Bournemouth Questionnaire score for bio-psycho-social im pairment diminished by about $25 \%$ within the first 12 months after the start of chiropractic treatment.

Back pain is a chronic disease for most, with episodes at short or long intervals. A preventive approach [to back pain] such as Maintenance Care makes sense. It is still not known if it 'works' because of the treatment given or because of the clinical encounter, or how these two components interact. towards SAM and a greater level of involvement 
Table 1 Aims and conclusions of papers published in the series What is chiropractic? (Continued)

\begin{tabular}{|c|c|c|}
\hline First author; year; title; study design & Aim of paper & Conclusion \\
\hline $\begin{array}{l}\text { Gislason et al. } 2019 . \\
\text { The shape of chiropractic in Europe: a cross } \\
\text { sectional survey of chiropractor's beliefs and } \\
\text { practice. } \\
\text { Cross-sectional survey }\end{array}$ & $\begin{array}{l}\text { To repeat a Canadian study within Europe } \\
\text { aiming at categorizing beliefs or potential } \\
\text { association with unorthodox practice. }\end{array}$ & $\begin{array}{l}\text { Around one fifth of European chiropractors } \\
\text { completing this survey identified themselves } \\
\text { with an a priori defined unorthodox description } \\
\text { of chiropractic care, i.e. "treating vertebral } \\
\text { subluxations as an encumbrance to the } \\
\text { expression of health". The data also showed a } \\
\text { number of key predictive practice behaviors } \\
\text { statistically associated. } \\
\text { with this unorthodox group, including higher } \\
\text { use of x-rays, higher patient visits, absence of } \\
\text { differential diagnostic approaches and less fa } \\
\text { vorable views regarding } \\
\text { the benefits of vaccination. }\end{array}$ \\
\hline
\end{tabular}

Papers describing new initiatives and developments in the chiropractic profession

Adams et al. 2018.

Leadership and capacity building in international chiropractic research: introducing the chiropractic academy for research leadership (CARL).

Commentary

Côté et al. 2019.

The development of a global chiropractic rehabilitation competency framework by the World Federation of Chiropractic.

Commentary

Discussion and debate papers

Coulter et al. 2019.

The research crisis in American institutions of complementary and integrative health: one proposed solution for chiropractic profession. Commentary

Leboeuf-Yde et al. 2019.

Chiropractic, one big unhappy family: better together or apart?

Commentary

Strahinjevich and Simpson 2018.

The schism in chiropractic through the eyes of a 1 st year chiropractic student.

Commentary can be healed.
To describe the aims, core principles, methodology and evolution of the Chiropractic Academy of Research Leadership (CARL).

To present a chiropractic rehabilitation competency framework developed by the World Federation of Chiropractic Disability and Rehabilitation Committee.

To discuss the overall crisis in research in complementary and alternative medicine and propose solutions with a special focus on chiropractic.
The long-term aim of CARL is to develop essen tial leadership skills and experiences to take on senior CARL mentorship appointments and help secure the successful mentorship of a subse quent early-career researcher cohort of Fellows.

Presents a chiropractic rehabilitation framework consisting of three domains: basic concepts of rehabilitation and disability; legal, regulatory and ethical components; and, rehabilitation management of disability and other health conditions.

If the institutions pool their limited talent and resources, they might be able to compete, but history has shown that the American chiropractic institutions have not yet been able to do that. However, perhaps through a mediator it might be achievable. We are not suggesting this proposal will solve all the problems and we recognize that outside of the US the situation is different. But within the US it seems to us that without some type of response the situation will get worse not better. The RAND Health program has signed onto this proposal and we are currently approaching foundations to fund it.

To examine the chiropractic profession from the perspective of an unhappy marriage by defining key elements in happy and unhappy marriages and by identifying factors that may determine why couples stay together or spilt up.

To review the historical origins of the schism in chiropractic and examine the influence the schism has had on the profession and to discuss a possible strategy whereby this schism
Chiropractors and chiropractic leaders, regardless of values and persuasion, need to pause and consider, if they are able to live and develop as they would like to in this centuryold unhappy marriage.

The schism as it is known has been identified as a division between those who adhere to the dogma of Palmerian ideology and those who embrace scientific advancement. Also considered were some of the reasons, largely external to the profession for the perpetuation of the divide. problem, most commonly low back pain (50\%) and neck pain $(23 \%)$, is female, middle-aged and employed [4]. Although there is some variation in practice styles, most people seeking care from chiropractors will receive spinal manipulation with around one third also receiving patient education, exercises and soft-tissue therapy [4].
There is an increasing body of literature describing chiropractors in interprofessional collaborations. Mior et al. reviewed literature dealing with chiropractors functioning in the health services of armed forces mainly from the US, Canada and Australia [5]. Stochkendahl et al. found that in the Scandinavian context, chiropractors can fulfil a key role in management of sickness absence and in work 
disability prevention both informally and as part of system-supported pathways [6,7]. Another example of chiropractors functioning in interprofessional collaboration was provided by Wirth et al. who reported on outcomes and trajectories of patients referred from a surgical department to chiropractic care [8]. In these settings, chiropractors frequently work alongside or in collaboration with other providers, and Salsbury et al. reported, based on a qualitative stakeholder analysis, that in order to be successful in multidisciplinary teams, chiropractors should be good clinicians, practice according to evidence, be communicative and be supportive of patient's emotional journeys during care and rehabilitation [9].

Four articles dealt with different elements of chiropractic practice. Axén et al. systematically reviewed evidence pertaining to chiropractic maintenance care and concluded that significant advances had been made in recent decades regarding what maintenance care is, when it should be applied, and what outcomes to expect [10]. Jenkins et al. reviewed practice guidelines dealing with use of spinal $x$-ray in chiropractic practice and concluded that routine use of $x$-ray is not in accordance with current evidence and practice guidelines [11]. Hansen et al. reported that chiropractors (at least in Denmark), when surveyed, commonly reported workrelated physical acute or overuse injuries in the low back, wrist, thumb and shoulder, but were very satisfied with their job and had a good psychosocial work environment [12]. Gíslason et al., in a survey of chiropractors in Europe, showed that around one fifth identified themselves with an "unorthodox" description of chiropractic care, and that this group were associated with a higher use of $\mathrm{x}$-rays, higher patient visits, absence of differential diagnostic approaches, and less favorable views regarding the benefits of vaccination [13].

Two papers reported on issues relating to education of chiropractors. Innes et al. compared accreditation standards from the Councils of Chiropractic Education between 2010 and 2016 and found that while there had been a move towards a focus on musculoskeletal care, mention of competencies in appraising scientific evidence and evidence-based practice had been removed from the criteria [14]. Funk et al. searched websites of 46 chiropractic educational programs in order to identify and count the mention of the term subluxation, and found that it is much more prevalent in descriptions of US-based educational institutions, and in fact the mention of subluxation had increased between 2011 and 2017 [15].

Papers describing new initiatives and developments in the chiropractic profession

Adams et al. described the aims, core principles, and methodology for the Chiropractic Academy for Research
Leadership (CARL). CARL aims to bring together a global network of early career researchers in chiropractic in a global network [16]. Côté et al. described a chiropractic rehabilitation competency framework that was developed by the World Federation of Chiropractic Disability and rehabilitation Committee [17].

\section{Discussion and debate papers}

The final group of papers centered on debates and discussions within chiropractic and their implications for professional unity and legitimacy. Coulter et al. described and discussed the research crisis in research in complementary and alternative medicine, with a focus on chiropractic, and suggested ways to advance this research endeavor [18]. Leboeuf-Yde et al. compared the chiropractic profession with an unhappy marriage between practitioners on a continuum from evidencebased, with a focus on management of musculoskeletal disorders, to conservative and vitalistic, with a focus on addressing subluxations to influence the nervous system. These authors suggested that it may be time to openly discuss separation or divorce so that the two fractions can pursue a separate future [19]. Strahinjevich and Simpson also targeted the schism between the evidenceoriented and the vitalistic groups in chiropractic as seen through the eyes of a first-year student, and discussed both the historic background for this and possible ways forward [20].

\section{Conclusions}

This commentary brings the 2017-2019 series What is Chiropractic? to a close. The series will remain open to including future articles, if relevant; this commentary provides a summary of the articles officially included in the series that have been published to date. When we made this call for papers in forming the thematic series, we were optimistic that we would energize the researchers in the profession to answer this question. The 18 papers have contributed significantly to a better understanding of what chiropractic is, where chiropractors practice and function, who seeks their care, what chiropractors do, and how they interact with other healthcare professionals. The series has not contributed with new data on effectiveness of the interventions chiropractors routinely apply to their patients; however, a casual search in PubMed using the search terms chiropractic or chiropractor for 2018 and 2019 identified over 700 papers, so clearly many scientific papers pertaining to chiropractic have been published in this and other journals alongside this series. Chiropractic and Manual Therapies is an open access journal, and papers in the series have been read widely with over 63,000 downloads (range 978 [18] - 15,000 [19]) and significant activity on social media. 
Papers in this series have again revealed deeply rooted disagreements within the chiropractic profession about what chiropractic is, and what it should be, as a profession $[13,19,20]$, as well as disagreements and variation in relation to education of chiropractors $[14,15]$ and chiropractic clinical practice [11]. In our opinion, it is ironic that while chiropractic has a strong presence in large parts of the world [3], is taking on increasingly important roles in disability prevention $[6,7,17]$, in the military [5] and in interprofessional care [8] as well as growing research capacity [16], discussions about fundamental values and direction of the profession are unresolved. This unresolved issue creates confusion for stakeholders and threatens to impede professionalization and cultural authority. If chiropractors are to remain relevant in today's evidence-based healthcare environment, there is an urgent need to agree on, and further describe, what chiropractic is, what chiropractors do and importantly to provide evidence for value of these activities to patients and societies.

\section{Acknowledgements}

Professors Hartvigsen and French would like to thank all authors contributing to this thematic series. Your contribution is greatly valued.

\section{Authors' contributions}

$\mathrm{JH}$ drafted the first version of the manuscript. Both authors subsequently revised and contributed until consensus was reached regarding the final version. Both authors read and approved the final manuscript.

\section{Funding}

No funding received in support of this editorial.

\section{Availability of data and materials}

Not applicable.

\section{Ethics approval and consent to participate}

Not applicable.

\section{Consent for publication}

Both authors approved the manuscript before submission.

\section{Competing interests}

The authors declare that they have no competing interests.

\section{Author details}

'Department of Sports Science and Clinical Biomechanics, University of Southern Denmark, Campusvej 55, 5230 Odense M, Denmark. ${ }^{2}$ Nordic Institute of Chiropractic, Campusvej 55, 5230 Odense M, Denmark. ${ }^{3}$ Department of Chiropractic, Macquarie University, Sydney, NSW 2109, Australia.

Received: 20 November 2019 Accepted: 26 December 2019 Published online: 30 January 2020

\section{References}

1. Hartvigsen J, French S. What is chiropractic? Chiropr Man Ther. 2017;25:30.

2. World Federation of Chiropractic. Definition of Chiropractic. 2001 https:// www.wfc.org/website/index.php?option=com_content\&view=article\&id= 90\&ltemid=110\&lang=en. Accessed 18 Dec 2019.

3. Stochkendahl MJ, Rezai M, Torres P, Sutton D, Tuchin P, Brown R, et al. The chiropractic workforce: a global review. Chiropr Man Ther. 2019;27:36.

4. Beliveau PJH, Wong JJ, Sutton DA, Simon NB, Bussieres AE, Mior SA, et al. The chiropractic profession: a scoping review of utilization rates, reasons for seeking care, patient profiles, and care provided. Chiropr Man Ther. 2017;25:35.
5. Mior S, Sutton D, Daphne T, Cancelliere C, French S, Taylor-Vaisey A, et al. Chiropractic services in the active duty military setting: a scoping review. Chiropr Man Ther. 2019;27:45.

6. Stochkendahl MJ, Larsen OK, Nim CG, Axen I, Haraldsson J, Kvammen OC, et al. Can chiropractors contribute to work disability prevention through sickness absence management for musculoskeletal disorders? - a comparative qualitative case study in the Scandinavian context. Chiropr Man Ther. 2018;26:15.

7. Stochkendahl MJ, Nim CG, Boyle E, Larsen OK, Axen I, Kvammen OC, et al. Managing sickness absence of patients with musculoskeletal pain - a crosssectional survey of Scandinavian chiropractors. Chiropr Man Ther. 2019;27:1.

8. Wirth B, Riner F, Peterson C, Humphreys BK, Farshad M, Becker S, et al. An observational study on trajectories and outcomes of chronic low back pain patients referred from a spine surgery division for chiropractic treatment. Chiropr Man Ther. 2019:27:6.

9. Salsbury SA, Vining RD, Gosselin D, Goertz CM. Be good, communicate, and collaborate: a qualitative analysis of stakeholder perspectives on adding a chiropractor to the multidisciplinary rehabilitation team. Chiropr Man Ther. 2018;26:29.

10. Axen I, Hestbaek L, Leboeuf-Yde C. Chiropractic maintenance care - what's new? A systematic review of the literature. Chiropr Man Ther. 2019;27:9.

11. Jenkins HJ, Downie AS, Moore CS, French SD. Current evidence for spinal Xray use in the chiropractic profession: a narrative review. Chiropr Man Ther. 2018;26:48.

12. Hansen MC, Aagaard T, Christensen HW, Hartvigsen J. Work-related acute physical injuries, chronic overuse complaints, and the psychosocial work environment in Danish primary care chiropractic practice - a cross-sectional study. Chiropr Man Ther. 2018;26:4

13. Gislason HF, Salminen JK, Sandhaugen L, Storbraten AS, Versloot R, Roug I, et al. The shape of chiropractic in Europe: a cross sectional survey of chiropractor's beliefs and practice. Chiropr Man Ther. 2019;27:16.

14. Innes SI, Leboeuf-Yde C, Walker BF. Comparing the old to the new: A comparison of similarities and differences of the accreditation standards of the chiropractic council on education-international from 2010 to 2016. Chiroprac Man Ther. 2018;26:25.

15. Funk MF, Frisina-Deyo AJ, Mirtz TA, Perle SM. The prevalence of the term subluxation in chiropractic degree program curricula throughout the world. Chiropr Man Ther. 2018;26:24.

16. Adams J, Kawchuk G, Breen A, De Carvalho D, Eklund A, Fernandez M, et al. Leadership and capacity building in international chiropractic research: introducing the chiropractic academy for research leadership (CARL). Chiropr Man Ther. 2018;26:5

17. Cote P, Sutton D, Nicol R, Brown R, Mior S. The development of a global chiropractic rehabilitation competency framework by the world Federation of Chiropractic. Chiropr Man Ther. 2019;27:29.

18. Coulter ID, Herman PM. The research crisis in American institutions of complementary and integrative health: one proposed solution for chiropractic profession. Chiropr Man Ther. 2019;27:32.

19. Leboeuf-Yde C, Innes SI, Young K, Kawchuk GN, Hartvigsen J. Chiropractic, one big unhappy family: better together or apart? Chiropr Man Ther. 2019;27:4.

20. Strahinjevich B, Simpson JK. The schism in chiropractic through the eyes of a 1st year chiropractic student. Chiropr Man Ther. 2018:26:2.

\section{Publisher's Note}

Springer Nature remains neutral with regard to jurisdictional claims in published maps and institutional affiliations.

Ready to submit your research? Choose BMC and benefit from:

- fast, convenient online submission

- thorough peer review by experienced researchers in your field

- rapid publication on acceptance

- support for research data, including large and complex data types

- gold Open Access which fosters wider collaboration and increased citations

- maximum visibility for your research: over $100 \mathrm{M}$ website views per year

At BMC, research is always in progress.

Learn more biomedcentral.com/submission 